PROCEEDINGS OF THE AMERICAN MATHEMATICAL SOCIETY

Volume 128, Number 1, Pages 183-188

S 0002-9939(99)05057-1

Article electronically published on July 1, 1999

\title{
A ZERO ENTROPY $T$ SUCH THAT THE [T,Id] ENDOMORPHISM IS NONSTANDARD
}

\author{
CHRISTOPHER HOFFMAN
}

(Communicated by Mary Rees)

\begin{abstract}
We present an example of an ergodic transformation $T$, a variant of a zero entropy non-loosely Bernoulli map of Feldman, such that the sequence of random variables generated by the $[T, \mathrm{Id}]$ endomorphism is nonstandard.
\end{abstract}

\section{INTRODUCTION}

Any sequence of random variables, $Y_{0}, Y_{1}, Y_{2}, \ldots$, defined on the space $Y$ produces a decreasing sequence of $\sigma$-algebras $\mathcal{F}_{n}$. These are defined by $\mathcal{F}_{n}=\sigma\left(Y_{n}, Y_{n+1}, \ldots\right)$. The sequence $Y_{i}$ is called exact if $\bigcap \mathcal{F}_{n}=\emptyset$. An isomorphism between two such sequences $\mathcal{F}_{n}$ and $\mathcal{G}_{n}$ is a 1-1, invertible, measure preserving map $\phi: \mathcal{F}_{0} \rightarrow \mathcal{G}_{0}$ such that $\phi\left(\mathcal{F}_{n}\right)=\mathcal{G}_{n} \forall n$. A sequence of random variables $Y_{i}$ is called standard if there exists an independent sequence of random variables $X_{i}$ producing an isomorphic sequence of $\sigma$-algebras. An equivalent definition is that there exists a sequence of independent partitions $\mathcal{I}_{n}$ such that $\mathcal{F}_{n}=\bigvee_{i=n}^{\infty} \mathcal{I}_{i}$. An endomorphism is said to be standard if the sequence of random variables produced by the endomorphism and a generating partition is standard.

Let $T$ be any 1-1 map on $(Y, \mathcal{C}, \nu)$. Let $S$ be the shift on $(X, \mathcal{B}, \mu)=\left(\{0,1\}^{\mathbb{N}}\right.$, Borel $\sigma$-algebra, product measure $\left.\left(\frac{1}{2}, \frac{1}{2}\right)\right)$. Define $[T$,Id $]$ on $(X \times Y, \mathcal{F}, \mu \times \nu)$ where $\mathcal{F}=\mathcal{B} \times \mathcal{C}$ by $[T, \operatorname{Id}](x, y)=\left(S x, T^{x_{0}} y\right)$. [T, Id $]$ is $2-1$, since any point $(x, y)$ has the preimages $(0 x, y)$ and $\left(1 x, T^{-1} y\right)$. Mejilson proved that $[T$,Id $]$ is exact whenever $T$ is ergodic $[6]$.

This paper gives an example of a zero entropy $T$, which is a variant of a zero entropy non-loosely Bernoulli transformation of J. Feldman [1]. The corresponding $[T$,Id] endomorphism is nonstandard. The arguments in this paper can easily be modified to show that for this $T$ the $\left[T, T^{-1}\right]$ endomorphism is nonstandard.

In [4] Heicklen and Hoffman proved that whenever $T$ has positive entropy then the $\left[T, T^{-1}\right]$ endomorphism, which has an analogous definition to the $[T, I d]$ endomorphism, is nonstandard. Feldman and Rudolph proved in [2] that whenever $T$ is rank 1 then $[T, I d]$ is standard. This argument can easily be modified to show that if $T$ is rank 1 and $T^{2}$ is ergodic then the $\left[T, T^{-1}\right]$ endomorphism is standard. The present paper shows that $T$ having positive entropy is not the only obstruction for $\left[T, T^{-1}\right]$ being standard.

Received by the editors June 17, 1997 and, in revised form, March 13, 1998.

1991 Mathematics Subject Classification. Primary 28D99; Secondary 60A10.

Key words and phrases. Decreasing sequences of $\sigma$-algebras, standard.

(C)1999 by Christopher Hoffman 
In [5] Heicklen, Hoffman, and Rudolph showed a connection between between the $\bar{f}$ metric, which is involved in the definition of loosely Bernoulli, and the $\bar{v}$ metric, which is involved in the criteria for standardness. By using a non loosely Bernoulli transformation to create a nonstandard $[T, \mathrm{Id}]$ endomorphism, the present paper strengthens this connection.

\section{STANDARDNESS CRITERIA}

In this section we introduce the terminology necessary to state the standardness criteria. An $\mathbf{n}$ branch is an element of $\{0,1\}^{n}$. An $\mathbf{n}$ tree is a binary tree of height $n$ consisting of $2^{n}$ branches. The top level is $b_{0}$ and the bottom level is $b_{n-1}$. Let $\mathcal{A}_{n}$ be the set of automorphisms of an $n$ tree.

For $m \leq n$ an $\mathbf{m}$ tree inside an $\mathbf{n}$ tree is a tree with $2^{m}$ branches such that the first $n-m$ coordinates all agree and the last $m$ coordinates vary over all possibilities. A labeled $\mathbf{n}$ tree for a partition $P$ over a point $y \in Y$ assigns to each branch $b$ the label $P\left(T^{\sum b_{i}} y\right)$. The Hamming metric on labeled $n$ trees is given by

$$
d_{n}\left(W, W^{\prime}\right)=\frac{\# \text { of branches where the labels of } W \text { and } W^{\prime} \text { disagree }}{2^{n}} .
$$

Fix $P$ and let $W$ and $W^{\prime}$ be labeled $n$ trees over $y$ and $y^{\prime}$ respectively. Define

$$
v_{n}^{P}\left(y, y^{\prime}\right)=\inf _{a \in \mathcal{A}_{n}} d_{n}\left(a W, W^{\prime}\right) .
$$

In the case that $\mathcal{F}_{n}$ comes from [T,Id], Vershik's standardness criterion is the following.

Theorem 2.1 (Vershik [7]). $\mathcal{F}_{n}$ is standard if and only if for every finite partition $P, \int v_{n}^{P}\left(y, y^{\prime}\right) d(\nu \times \nu) \rightarrow 0$.

Remark 2.1. A possibly more accessible proof of this can be found in [3].

We also present some terminology used in the proof. A branch $b$ in the tree over $y$ lands at a point $\mathbf{z}$ if $z=T^{\sum b_{i}} y$. A subtree of height $k$ in the $n$ tree over $y$ lies over a point $\mathbf{z}$ if for any branch $b$ in the subtree $z=T^{\sum_{0}^{n-k-1} b_{i}} y$.

\section{Description OF $T$}

As we said in the introduction the transformation $T$ will be a variant of one constructed by J. Feldman [1]. His example showed that there are ergodic transformations which are not loosely Bernoulli. The ergodic transformation presented below is also not loosely Bernoulli. The proof of this is the same as the proof for Feldman's transformation.

Our space is a subset of $\Omega=\left\{a_{1}, a_{2}, \ldots, a_{100}\right\}^{\mathbf{Z}}$ which is defined by a cutting and stacking procedure. The partition $P$ is the partition into 100 sets depending on the symbol $y_{0}$. There exist 100 different 1 blocks, each of length 1 , with $B_{1}^{i}=a_{i}$. For every $n$ each $n$ block has the same measure. 
Now suppose we have $n-1$ blocks, $B_{n-1}^{1}, B_{n-1}^{2}, \ldots, B_{n-1}^{N(n-1)}$. We construct $N(n)=n^{10}$ different $n$ blocks in the following way:

$$
\begin{aligned}
& B_{n}^{1}=\left[\left(B_{n-1}^{1}\right)\left(B_{n-1}^{2}\right) \ldots\left(B_{n-1}^{N(n-1)}\right)\right]^{\left(n^{10}\right)^{\left(n^{10}\right)}}, \\
& B_{n}^{2}=\left[\left(B_{n-1}^{1}\right)^{n^{10}}\left(B_{n-1}^{2}\right)^{n^{10}} \cdots\left(B_{n-1}^{N(n-1)}\right)^{n^{10}}\right]^{\left(n^{10}\right)^{\left(n^{10}-1\right)}}, \\
& B_{n}^{i}=\left[\left(B_{n-1}^{1}\right)^{\left(n^{10}\right)^{i-1}}\left(B_{n-1}^{2}\right)^{\left(n^{10}\right)^{i-1}} \cdots\left(B_{n-1}^{N(n-1)}\right)^{\left(n^{10}\right)^{i-1}}\right]^{\left(n^{10}\right)^{\left(n^{10}-i+1\right)}}, \\
& B_{n}^{N(n)}=\left[\left(B_{n-1}^{1}\right)^{\left(n^{10}\right)^{\left(n^{10}-1\right)}}\left(B_{n-1}^{2}\right)^{\left(n^{10}\right)^{\left(n^{10}-1\right)}} \cdots\left(B_{n-1}^{N(n-1)}\right)^{\left(n^{10}\right)^{\left(n^{10}-1\right)}}\right]^{\left(n^{10}\right)} .
\end{aligned}
$$

Thus at stage $n$ we have $N(n)=n^{10}$ different $n$ blocks, each of length $l(n)=$ $l(n-1) N(n-1)\left(n^{10}\right)^{\left(n^{10}\right)}$. $T$ is the shift.

We will make one definition about the block structure of $T$ which is important in the next section. An $n$ region in an $n$ block $B_{n}^{i}$ is a maximal subword in the $n$ block which consists of one $n-1$ block repeated many times. The length of an $n$ region in an $n$ block $B_{n}^{i}$ is $l(n-1)\left(n^{10}\right)^{i-1}$.

\section{4. $[T$, ID $]$ IS NONSTANDARD}

The proof is by induction. Excluding small sets the argument is as follows. We will define a sequence of heights $h_{n}$ increasing to infinity and a sequence $\epsilon_{n}$ which converges down to $\epsilon>0$. We will show that for $y$ in $n$ block $B_{j}^{i}$, $y^{\prime}$ in $n$ block $B_{n}^{j}$ with $j>i$ then $v_{h_{n}}\left(y, y^{\prime}\right)>\epsilon_{n}$. This statement will be enough to show that [T,Id] is nonstandard. We show this by defining a height $k_{n}=k_{n}^{i, j}$ so that $h_{n-1}$ subtrees of a $k_{n}$ subtree of the $h_{n}$ tree over $y$ lie over many different $n-1$ blocks. But $h_{n-1}$ subtrees of a $k_{n}$ subtree over $y^{\prime}$ lie over only one $n-1$ block. A tree automorphism must take $k_{n}$ subtrees to $k_{n}$ subtrees, and $h_{n-1}$ subtrees to $h_{n-1}$ subtrees. By the inductive hypothesis the $v_{h_{n-1}}$ distance between most of the $h_{n-1}$ subtrees paired by a tree automorphism is greater than $\epsilon_{n-1}$ so the $v_{h_{n}}$ distance between the $h_{n}$ trees is greater than $\epsilon_{n}$.

In order to do the base case of the induction we need the following lemma.

Lemma 4.1. For any $y=y_{0}, \ldots, y_{h-1}$, such that $y$ is not of period 2 , then the only other point $y^{\prime}$ such that $v_{h}\left(y, y^{\prime}\right)=0$ is $y_{i}^{\prime}=y_{h-1-i}$.

Proof. A proof of this lemma is contained in [4].

At various times we will use the following fact about binomial distributions.

Lemma 4.2. Given $n$ and $k$, define $p_{i}=\sum_{j=i \bmod k}\left(\begin{array}{c}n \\ j\end{array}\right)$. Then

$$
\sum_{i=0}^{k-1}\left|\frac{p_{i}}{2^{n}}-\frac{1}{k}\right| \leq \frac{2 k}{\sqrt{n}}
$$

Proof. The binomial $n$ distribution and another copy of it translated by $k$ differ by at most $\frac{k 2^{n}}{\sqrt{n}}$, because the binomial coefficients are less than $\frac{2^{n}}{\sqrt{n}}$. Take the $i$ for which $p_{i}$ is the lowest. Define the distribution $D(j)=\left(\begin{array}{l}n \\ l\end{array}\right)$ where $l$ is the greatest integer $\leq j$ and $l=i \bmod k$. This distribution lies in between the two distributions 
mentioned above. Thus the sum of absolute value of the terms which are negative is at most $\frac{k}{\sqrt{n}}$. A similar argument bounds the sum of the positive terms.

Before we get to the main part of the induction we must choose our parameters and make another definition. Choose $h_{n}$ so that

$$
\sqrt{h_{n}}=(l(n-1))\left(n^{10}\right)^{\left(n^{10}-1\right)}
$$

is the length of the longest $n$ region. Define

$$
\epsilon_{2}=1 /\left(2^{h_{2}}+1\right) \text { and } \epsilon_{n}=\epsilon_{n-1}\left(1-100 n^{-2}\right) .
$$

As $\sum 100 n^{-2}<\infty, \lim \epsilon_{n}=\epsilon>0$.

There is one last definition that we will use heavily. We say that a tree of height $h$ over a point $y$ falls mostly in an $n$ block (or $n$ region) if the interval $(h / 2-n \sqrt{h}, h / 2+n \sqrt{h})$ is entirely in one $n$ block (region) in $y$. If an $h_{n-1}$ subtree of the $h_{n}$ tree over $y$ or $y^{\prime}$ falls mostly in $B_{n-1}^{m}$, for some $m$, then label that subtree $m$. An application of Lemma 4.2 shows that most $h_{n-1}$ subtrees of a $k_{n}$ subtree over any point fall mostly in one $n-1$ block and thus receive a label. This fraction is at least

$$
1-\frac{2 l(n-1)}{\sqrt{k_{n}}}-\frac{2 n \sqrt{h_{n-1}}}{l(n-1)}>1-4 n^{-2} .
$$

Now choose $k_{n}^{i, j}=k_{n}$ so that

$$
\sqrt{k_{n}}=l(n-1)\left(n^{10}\right)^{i-1 / 2} .
$$

This length $\sqrt{k_{n}}$ is between the length of the $n$ regions in $B_{n}^{i}$ and the length of the $n$ region in $B_{n}^{j}$. This will imply that most $k_{n}$ subtrees in the $h_{n}$ tree over $y$ have many different labels on their $h_{n-1}$ subtrees. But most $k_{n}$ subtrees in the $h_{n}$ tree over $y^{\prime}$ have essentially only one type of labeled $h_{n-1}$ subtree. The next lemma will prove the first of these assertions and the second lemma will prove the latter. The combination of the two will form the heart of the inductive step.

Lemma 4.3. If the $h_{n}$ tree over y falls mostly over $n$ block $B_{n}^{i}$, then for a fraction $1-2 n^{-2}$ of the $k_{n}$ subtrees in the $h_{n}$ tree over $y$, and for any given label, a fraction at most $3 n^{-2}$ of the $h_{n-1}$ subtrees have that label.

Proof. We will use an estimate on the binomial coefficients to show that $k_{n}$ subtrees of the $h_{n}$ tree over $y$ which fall mostly in $n$ block $B_{n}^{i}$ have many different labels on their $h_{n-1}$ subtrees. None of these labels have a large fraction of the $h_{n-1}$ subtrees with that label. First we show that only a small fraction of the branches of a $k_{n}$ tree land in any given $n-1$ region in $n$ block $B_{n}^{i}$. Then we use that to show that only a small fraction of $h_{n-1}$ blocks in a $k_{n}$ tree can have the same label. No binomial coefficient is larger than $2^{n} / \sqrt{n}$. So the number of branches of a $k_{n}$ tree that land in a given $h_{n-1}$ region is at most $2^{k_{n}} / \sqrt{k_{n}}$ multiplied by the length of an $n$ region in block $B_{n}^{i}$. Since $\sqrt{k_{n}}$ is much larger than $l(n-1)\left(n^{10}\right)^{i-1}$, the length of an $n$ region in block $B_{n}^{i}$, the fraction of branches that land in a given $h_{n-1}$ region is less than $n^{-2}$.

The distance between two $h_{n-1}$ regions in block $B_{n}^{i}$ that are made out of the same $n-1$ block is $l(n-1)\left(n^{10}\right)^{i-1}\left(n^{10}-1\right)$. Since this is far larger than $\sqrt{k_{n}}$ a Chebychev estimate shows that if the number of branches in one $h_{n-1}$ region has close to the bound then the number of branches that land in all other $h_{n-1}$ regions that are made out of the same $n-1$ block is miniscule. So the fraction of branches 
that land in a given $h_{n-1}$ block is less than $2 n^{-2}$. The fraction of $h_{n-1}$ subtrees with a given label is then less than $3 n^{-2}$. Finally a Chebychev estimate shows that the fraction of $k_{n}$ subtrees to which the preceding argument applies, those which fall mostly in $n$ block $B_{n}^{i}$, is at least $1-2 n^{-2}$ of all the $k_{n}$ subtrees.

For the $k_{n}$ subtrees over $y^{\prime}$ the picture is completely different. Most $k_{n}$ subtrees of the $h_{n}$ tree over $y^{\prime}$ have the same label on almost all of their $h_{n-1}$ subtrees.

Lemma 4.4. If the $h_{n}$ tree over $y^{\prime}$ falls mostly over $n$ block $B_{n}^{j}$, then for a fraction $1-6 n^{-2}$ of the $k_{n}$ subtrees in the $h_{n}$ tree over $y^{\prime}$ a fraction at least $1-2 n^{-2}$ of the $h_{n-1}$ subtrees in one of these $k_{n}$ subtrees have the same label.

Proof. As in the last lemma the $k_{n}$ subtrees which fall mostly in $n$ block $B_{n}^{j}$ are at least $1-2 n^{-2}$ of all the $k_{n}$ subtrees. Of these subtrees, Lemma 4.2 implies those that do fall mostly in an $n$ region in block $B_{n}^{j}$ make up a fraction at least

$$
1-\frac{2 n \sqrt{k_{n}}}{l(n-1)\left(n^{10}\right)^{j-1}}-\frac{2 l(n-1)\left(n^{10}\right)^{j-1}}{\sqrt{h_{n}}}>1-4 n^{-2} .
$$

In such a $k_{n}$ subtree, a Chebychev estimate shows that a fraction at least $1-2 n^{-2}$ of the $h_{n-1}$ subtrees have the same label. Thus in $1-6 n^{-2}$ of the $k_{n}$ subtrees in the big tree over $y^{\prime}$ a fraction at least $1-2 n^{-2}$ of the $h_{n-1}$ subtrees have the same label.

Theorem 4.1. The $[T, \mathrm{Id}]$ endomorphism is nonstandard.

Proof. We will prove the following inductive statement. If the $h_{n}$ tree over $y$ falls mostly in $n$ block $B_{n}^{i}$, the $h_{n}$ tree over $y^{\prime}$ falls mostly in $n$ block $B_{n}^{j}$, and $i \neq j$ then $v_{h_{n}}\left(y, y^{\prime}\right)>\epsilon_{n}$. This will complete the proof because $2 n \sqrt{h_{n}}$ is much smaller than $l(n)$ so most points $y$ have the $h_{n}$ over $y$ tree falling mostly in one $n$ block. In addition $h_{n} \rightarrow \infty$, and $\epsilon_{n} \rightarrow \epsilon>0$. Thus these facts, the induction, and Theorem 2.1 will imply that $[T, \mathrm{Id}]$ is nonstandard.

Case $n=2$ : Since $\epsilon_{2}=1 /\left(2^{h_{2}}+1\right)$ the two trees must match perfectly after some tree isomorphism. No word of length $h_{2}$ in one 2 block is identical to a word of length $h_{2}$ or its reflection in a different 2 block, or is of period 2, so Lemma 4.1 shows the base case is true.

Case $n$ : Assume the lemma is true for $n-1$ and that $i<j$, are given. A tree automorphism must take $k_{n}$ subtrees to $k_{n}$ subtrees, and $h_{n-1}$ subtrees to $h_{n-1}$ subtrees. By Lemma 4.4 the $k_{n}$ subtrees over $y^{\prime}$ have essentially only one type of labeled $h_{n-1}$ subtree. But by Lemma 4.3 the $k_{n}$ subtrees over $y$ have many different labels. Since most $h_{n-1}$ subtrees are labeled, most $h_{n-1}$ subtrees must be mapped to an $h_{n-1}$ subtree with a different label. On these $h_{n-1}$ subtrees the inductive hypothesis applies. Thus for any automorphism of the tree over $y$ we can apply the inductive hypothesis to a fraction at least $1-100 n^{-2}$ of the $h_{n-1}$ subtrees. As $\epsilon_{n}=\epsilon_{n-1}\left(1-100 n^{-2}\right)$ the inductive step is true. This completes the proof.

\section{REFERENCES}

[1] J. Feldman. New K-automorphisms and a problem of Kakutani. Israel J. Math. 24 (1976), no. 1, 16-38. MR 53:13515

[2] J. Feldman, D. Rudolph. Standardness of sequences of $\sigma$-fields given by certain endomorphisms. Fund. Math. 157 (1998), no. 2-3, 175-189. CMP 98:16

[3] D. Heicklen. Bernoullis are standard when entropy is not an obstruction. Israel J. Math. 107 (1998), 141-155. CMP 99:05 
[4] D. Heicklen, C. Hoffman. T,T $T^{-1}$ is not standard. ETDS. 18 (1998), no. 4, 875-878. CMP 99:01

[5] D. Heicklen, C. Hoffman, D. Rudolph. Entropy and dyadic equivalence of random walks on a random scenery. preprint.

[6] I. Mejilson. Mixing properties of a class of skew-products. Israel J. Math. 19 (1974), 266-270. MR 51:8374

[7] A. M. Vershik. Decreasing sequences of measurable partitions and their applications. Dokl. Akad. Nauk SSSR 193 no. 4 (1970), 748-751. MR 42:3258

The Hebrew University, Institute of Mathematics, Jerusalem, Israel

Current address: Department of Mathematics, University of Maryland, College Park, Maryland 20742

E-mail address: hoffman@math.umd.edu 